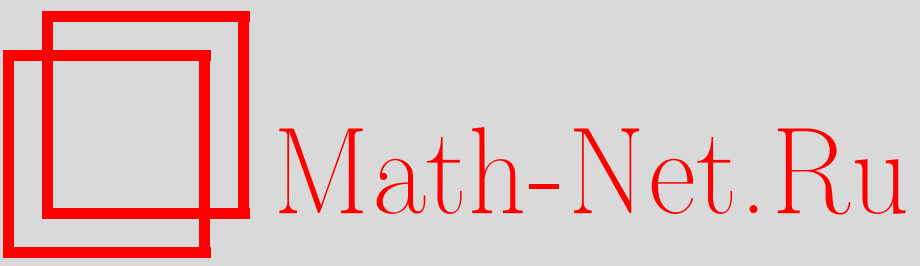

В. Ю. Ровенский, Проблемы внешней геометрии слоений, Итоги науки и техн. Сер. Соврем. мат. и ее прил. Темат. обз., 2020, том 179, 41-49

DOI: https://doi.org/10.36535/0233-6723-2020-179-41-49

Использование Общероссийского математического портала Math-Net.Ru подразумевает, что вы прочитали и согласны с пользовательским соглашением

http://www.mathnet.ru/rus/agreement

Параметры загрузки:

IP: 54.224 .187 .69

26 апреля 2023 г., 18:23:57 


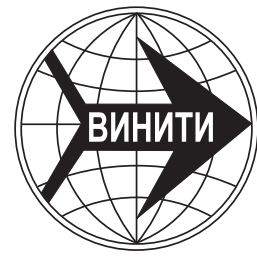

ИТОГИ НАУКИ И ТЕХНИКИ.

Современная математика и ее приложения.

Тематические обзоры.

Том 179 (2020). C. $41-49$

DOI: $10.36535 / 0233-6723-2020-179-41-49$

УДК 514.76

\title{
ПРОБЛЕМЫ ВНЕШНЕЙ ГЕОМЕТРИИ СЛОЕНИЙ
}

\author{
(c) 2020 г. $\quad$ В. Ю. РОВЕНСКИЙ
}

\begin{abstract}
АннотАция. Данный обзор посвящен частным проблемам внешней геометрии слоений, которая, грубо говоря, описывает, как слои (или отдельные подмногообразия) расположены внутри объемлющего псевдориманова пространства. Обсуждаются следующие темы, связанные со смешанной скалярной кривизной: интегральные формулы и расщепление слоений, предписывающие смешанную кривизну слоений, и вариации функционалов, определенных на слоениях, которые играют центральную роль во внешней геометрии.
\end{abstract}

Ключевые слова: внешняя геометрия, псевдориманова метрика, аффинная связность, слоение, смешанная скалярная кривизна, интегральная формула, вариация.

\section{PROBLEMS OF EXTRINSIC GEOMETRY OF FOLIATIONS}

\section{(c) 2020 V. YU. ROVENSKII}

\begin{abstract}
This survey is devoted to particular problems of extrinsic geometry of foliations, which, roughly speaking, describes how leaves (or single submanifolds) are located within the ambient pseudoRiemannian space. We discuss the following topics with the mixed scalar curvature: integral formulas and splitting of foliations, prescribing the mixed curvature of foliations, and variations of functionals defined on foliations, which seem to be central in extrinsic geometry.
\end{abstract}

Keywords and phrases: extrinsic geometry, pseudo-Riemannian metric, affine connection, foliation, mixed scalar curvature, integral formula, variation.

AMS Subject Classification: 53C12, 53C21

Введение. Слоения, т.е. разбиения многообразия на наборы подмногообразий одинаковой размерности, называемые листами, появились в 1940-х гг. в работах Г. Риба и К. Эресмана; с тех пор эта тема быстро развивалась (см., например, [6]). Смешаннал скалярная кривизна - простейший инвариант расслоенного псевдориманова многообразия или, в более общем смысле, многообразия, наделенного двумя невырожденными дополнительными ортогональными распределениями. Основными понятиями внешней геометрии являются операторы конуля распределений, вторые фундаментальные формы слоев в случае слоений, смешанная кривизна и их инварианты. Отметим, что пространство-время (4-мерное многообразие, снабженное лоренцевой метрикой и времениподобным единичным векторным полем, не обязательно интегрируемым нормальным распределением) имеет структуру почти произведения (два дополнительных ортогональных распределения); более того, глобально гиперболические (и стабильно причинные) пространства-времена естественным образом расслаиваются (см. [4]). Смешанная скалярная кривизна используется в следующих темах, которые, по-видимому, являются центральными во внешней геометрии (cм. [15]):

(i) интегральные формулы и расщепление слоений (см. $[8,10,13,14,21])$;

(ii) предписывание смешанной кривизны: задачи типа Ямабе (см. [18, 19]);

(iii) вариационные задачи: действие типа Эйнштейна-Гильберта (см. [3, 11, 12, 16, 17]), 
1. Предварительные сведения. В дальнейшем будем использовать следующие обозначения: $(M, g)$ - связное псевдориманово многообразие; $T M$ - касательное расслоение многообразия $M$; $\bar{\nabla}$ - линейная связность на $M$ с тензором кручения

$$
\mathcal{S}(X, Y)=\bar{\nabla}_{X} Y-\bar{\nabla}_{Y} X-\bar{\nabla}_{[X, Y]}(X, Y \in T M) ;
$$

$\mathfrak{T}:=\bar{\nabla}-\nabla$ - тензор разности, где $\nabla$ - связность Леви-Чивиты, согласованная с римановой метрикой $g$; тензор $\mathfrak{T}$ связан с тензором кручения формулой

$$
2 \mathcal{S}(X, Y)=\mathfrak{T}(X, Y)-\mathfrak{T}(Y, X) .
$$

Тензор кривизны $R$ связности $\bar{\nabla}$, тензор Риччи $\overline{\operatorname{Ric}}$ и скалярная кривизна $\overline{\mathrm{S}}$ равны соответственно

$$
\bar{R}_{X, Y}=\bar{\nabla}_{Y} \bar{\nabla}_{X}-\bar{\nabla}_{X} \bar{\nabla}_{Y}+\bar{\nabla}_{[X, Y]}, \quad \overline{\operatorname{Ric}}=\operatorname{Tr}_{1,3} \bar{R}, \quad \overline{\mathrm{S}}=\operatorname{Tr}_{g} \overline{\operatorname{Ric}} .
$$

Будем рассматривать следующие фундаментальные структуры на $(M, g)$ :

(i) риманова структура: $\bar{\nabla}=\nabla$;

(ii) структура Римана-Картана: $\bar{\nabla} g=0$;

(iii) статистическая структура: связность $\bar{\nabla}$ бз кручения, для которой тензор $\bar{\nabla} g$ симметричен.

Будем также рассматривать риманову структуру почти произведения на $(M, g)$, состоящую из $p$-мерного невырожденного распределения $\widetilde{\mathcal{D}}$ (подрасслоения касательного расслоения $T M$, возможно, касательного к слоению $\mathcal{F}$ ) и $n$-мерного распределения $\mathcal{D}, g$-ортогонального к $\widetilde{\mathcal{D}}$. Внешнял геометрия пары $(\mathcal{D}, \widetilde{\mathcal{D}})$ на $(M, g)$ определяется операторами конуля

$$
C_{U}(X)=-\left(\nabla_{X} U\right)^{\top}, \quad \tilde{C}_{X}(U)=-\left(\nabla_{U} X\right)^{\perp}
$$

где $X \in \Gamma(\widetilde{\mathcal{D}}), U \in \Gamma(\mathcal{D})$ и $(\cdot)^{\top},(\cdot)^{\perp}$-ортогональные проекции касательного расслоения $T M$ на $\widetilde{\mathcal{D}}$ и на $\mathcal{D}$. Введем понятие $\widetilde{\mathcal{D}}$-связанных тензоров $(\mathcal{D}$-связанные тензоры вводятся аналогично). Самосопряженный оператор формы $A_{U}$ и кососииметричный оператор $T_{U}^{\sharp}$ на $\widetilde{\mathcal{D}}$ дуальны второй фундаментальной форме $h=\frac{1}{2}\left(\nabla_{X} Y+\nabla_{Y} X\right)^{\perp}$ и тензору интегрируемости $T=\frac{1}{2}[X, Y]^{\perp}$ распределения $\widetilde{\mathcal{D}}$ :

$$
g\left(A_{U} X, Y\right)=\langle h(X, Y), U\rangle, \quad\left\langle T_{U}^{\sharp} X, Y\right\rangle=\langle T(X, Y), U\rangle, \quad X, Y \in \widetilde{\mathcal{D}}, U \in \mathcal{D} .
$$

Имеем $C_{U}=A_{U}+T_{U}^{\sharp}$; таким образом,

$$
A_{U}=\frac{1}{2}\left(C_{U}+C_{U}^{*}\right), \quad T_{U}^{\sharp}=\frac{1}{2}\left(C_{U}-C_{U}^{*}\right) .
$$

Распределение $\widetilde{\mathcal{D}}$ вполне геодезично, если $h=0$; гармонично, если $H:=\operatorname{Tr}_{g} h=0$ (H-вектор средней кривизны); вполне омбилично, если $h=\left.(H / n) g\right|_{\widetilde{\mathcal{D}}}$. Если распределение $\widetilde{\mathcal{D}}$ интегрируемо, т.е., $T=0$, то оно определяет некоторое слоение.

Смешанная секиионная кривизна $K_{\mathrm{mix}}$ - это секционная кривизна плоскостей, которые нетривиально пересекают каждое из распределений. Для вполне геодезического слоения $K_{\text {mix }}$ связана с уравнением Якоби $\ddot{U}+R(\dot{\gamma}, U) \dot{\gamma}=0$ на листовых геодезических $\gamma$. Смешанная секционная кривизна $K_{\text {mix }}$ связана также с такими понятиями, как кривизна и размерностъ вполне геодезического слоения (см. [9]) и римановы субмерсии.

Для диапазонов изменения индексов примем следующие соглашения:

$$
a, b, c \ldots \in\{1, \ldots, n\}, \quad i, j, k \ldots \in\{1, \ldots, p\} .
$$

Смешанная скалярная кривизна многообразия $(g, \nabla)$ - это усредненное значение $K_{\text {mix }}$,

$$
\mathrm{S}_{\text {mix }}=\mathrm{S}-\mathrm{S}_{\mathcal{D}}-\mathrm{S}_{\widetilde{\mathcal{D}}}=\sum_{a, i} \varepsilon_{a} \varepsilon_{i} K\left(E_{a}, \mathcal{E}_{i}\right),
$$


где $\left\{E_{a} \in \Gamma(\widetilde{\mathcal{D}}), \mathcal{E}_{i} \in \Gamma(\mathcal{D})\right\}$-локальный ортонормированный репер на $M, \varepsilon_{i}=\left\langle\mathcal{E}_{i}, \mathcal{E}_{i}\right\rangle= \pm 1$, $\varepsilon_{a}=\left\langle E_{a}, E_{a}\right\rangle= \pm 1$. Если $\widetilde{\mathcal{D}}$ (или $\mathcal{D}$ ) - линейная оболочка единичного векторного поля $N$, то $\mathrm{S}_{\mathrm{mix}}=\varepsilon_{N} \operatorname{Ric}_{N, N}$. Смешанная скалярная кривизна многообразия $(g, \bar{\nabla})$ задается формулой

$$
\overline{\mathrm{S}}_{\mathrm{mix}}=\frac{1}{2} \sum_{a, i} \varepsilon_{a} \varepsilon_{i}\left[\left\langle\bar{R}\left(E_{a}, \mathcal{E}_{i}\right) E_{a}, \mathcal{E}_{i}\right\rangle+\left\langle\bar{R}\left(\mathcal{E}_{i}, E_{a}\right) \mathcal{E}_{i}, E_{a}\right\rangle\right]
$$

Используя уравнение Риччи для распределений (см. [9])

$$
\langle R(U, X) V, Y\rangle=\left\langle\left(\left(\nabla_{U} C\right)_{V}-C_{V} C_{U}\right) X, Y\right\rangle+\left\langle\left(\left(\nabla_{X} \tilde{C}\right)_{Y}-\tilde{C}_{Y} \tilde{C}_{X}\right) U, V\right\rangle
$$

тензоры типа Казорати

$$
\widetilde{\mathcal{A}}=\sum_{a} \varepsilon_{a} \tilde{A}_{a}^{2}, \quad \widetilde{\mathcal{T}}=\sum_{a} \varepsilon_{a}\left(\tilde{T}_{a}^{\sharp}\right)^{2},
$$

и тензор деформации векторного поля $H$ вдоль $\mathcal{D}$, получим

$$
\operatorname{Ric}_{X, Y}^{\perp}=\operatorname{div}^{\top} \tilde{h}(X, Y)-\langle(\widetilde{\mathcal{A}}+\widetilde{\mathcal{T}})(X), Y\rangle+\operatorname{Def}_{\mathcal{D}} H(X, Y)-\operatorname{Tr}_{g}\left(C_{Y} C_{X}\right)
$$

здесь симметричный $(0,2)$-тензор

$$
\operatorname{Ric}_{X, Y}^{\perp}:=\sum_{a} \varepsilon_{a}\left\langle R\left(X^{\perp}, E_{a}\right) Y^{\perp}, E_{a}\right\rangle
$$

(см. [11]), относящийся к внешней геометрии, называется частной кривизной Риччи распределения $\widetilde{\mathcal{D}}$. Имеем $\operatorname{Tr}_{g} \operatorname{Ric}^{\perp}=\mathrm{S}_{\text {mix }}$; кроме того, используя (1), получаем следующую формулу (см. [21]):

$$
\mathrm{S}_{\text {mix }}=\operatorname{div}(H+\widetilde{H})-\|h\|^{2}+\|H\|^{2}-\|\tilde{h}\|^{2}+\|\widetilde{H}\|^{2}+\|T\|^{2}+\|\tilde{T}\|^{2} .
$$

2. Интегральные формулы и расщепление слоений. Интегральные формулы, будучи инструментом для получения глобальных результатов в геометрии, являются препятствиями для существования слоений (или их компактных слоев) с заданной внешней геометрией. Идея доказательства интегральных формул состоит в вычислении дивергенции векторного поля и последующем применении теоремы Стокса. Интегрируя (2) на замкнутом римановом многообразии $(M, g)$, получаем

$$
\int_{M}\left(\mathrm{~S}_{\text {mix }}-\|\tilde{T}\|^{2}-\|T\|^{2}+\|\tilde{h}\|^{2}+\|h\|^{2}-\|\tilde{H}\|-\|H\|^{2}\right) d \operatorname{vol}_{g}=0 .
$$

В частности, для вполне омбилического распределения $(\mathcal{D}, \widetilde{\mathcal{D}})$ из $(2)$ получаем

$$
\operatorname{div}(H+\widetilde{H})=\mathrm{S}_{\text {mix }}-\frac{p-1}{p}\|H\|^{2}-\frac{n-1}{n}\|\widetilde{H}\|^{2}-\|T\|^{2}-\|\tilde{T}\|^{2},
$$

откуда следует расщепление $\mathrm{S}_{\mathrm{mix}} \leqslant 0$. Для гармонических слоений имеем $0=\mathrm{S}_{\mathrm{mix}}+\|h\|^{2}+\|\tilde{h}\|^{2}$, так что получаем расщепление $\mathrm{S}_{\mathrm{mix}} \geqslant 0$.

Указанный подход можно применить к векторным полям $Z_{k}=\left(A_{H}\right)^{k} \widetilde{H}+\left(\tilde{A}_{\widetilde{H}}\right)^{k} H$ на римановых многообразиях при $k>0$ (см. [8]). В случае метрически-аффинных пространств добавим к $Z_{k}$, $k=0,1$, некоторые векторные поля и вычислим дивергенции (см. [13]). Положим $V=(\mathcal{D} \times \widetilde{\mathcal{D}}) \oplus$ $(\widetilde{\mathcal{D}} \times \mathcal{D})$ и определим $(1,2)$-тензоры $\mathfrak{T}^{*}$ и $\widehat{\mathfrak{T}}$ формулами

$$
\left\langle\mathfrak{T}_{X}^{*} Y, Z\right\rangle=\left\langle\mathfrak{T}_{X} Z, Y\right\rangle, \quad \widehat{\mathfrak{T}}_{X} Y=\mathfrak{T}_{Y} X,
$$

а также векторы типа средней кривизны

$$
H_{\mathfrak{T}}=\sum_{a} \varepsilon_{a} \mathfrak{T}_{a} E_{a}, \quad \widetilde{H}_{\mathfrak{T}}=\sum_{i} \varepsilon_{i} \mathfrak{T}_{i} \mathcal{E}_{i}
$$


Предложение 1 (см. [13]). Имеет место формула

$$
\begin{aligned}
& \operatorname{div}\left(\left(H_{\mathfrak{T}-\mathfrak{T}^{*}}\right)^{\perp}+\left(\widetilde{H}_{\mathfrak{T}-\mathfrak{T}^{*}}\right)^{\top}\right)=2\left(\overline{\mathrm{S}}_{\text {mix }}-\mathrm{S}_{\text {mix }}\right)-\left\langle H_{\mathfrak{T}}, \widetilde{H}_{\mathfrak{T}^{*}}\right\rangle-\left\langle\widetilde{H}_{\mathfrak{T}}, H_{\mathfrak{T}^{*}}\right\rangle- \\
& \quad-\left\langle H_{\mathfrak{T}-\mathfrak{T}^{*}}+\widetilde{H}_{\mathfrak{T}^{*}-\mathfrak{T}}, H-\widetilde{H}\right\rangle-\left\langle\mathfrak{T}-\mathfrak{T}^{*}+\widehat{\mathfrak{T}}-\widehat{\mathfrak{T}^{*}}, \tilde{A}-\tilde{T}^{\sharp}+A-T^{\sharp}\right\rangle+\left\langle\mathfrak{T}^{*}, \widehat{\mathfrak{T}}\right\rangle_{\mid V} .
\end{aligned}
$$

В частности, для статистических многообразий формула (3) имеет вид

$$
\overline{\mathrm{S}}_{\text {mix }}-\mathrm{S}_{\text {mix }}=\left\langle H_{\mathfrak{T}}, \widetilde{H}_{\mathfrak{T}}\right\rangle-\left.\frac{1}{2}\langle\mathfrak{T}, \mathfrak{T}\rangle\right|_{V} .
$$

Таким образом, приведенные выше интегральные формулы и результаты о расщеплении могут быть распространены на метрически-аффинные многообразия.

3. Предписание смешанной кривизны. Геометрические задачи о предписании кривизны многообразия с помощью конформной замены метрики, например, проблема задания гауссовой кривизны на замкнутой поверхности, известны давно (см. [2,7]). Рассмотрим задачу типа Ямабе (см. [19]) о существовании метрики постоянной смешанной кривизны $\overline{\mathrm{S}}_{\mathrm{mix}}=$ const на данном многообразии $\mathcal{D}$-конформной структуры $\left(g=g^{\top}+g^{\perp}, \mathfrak{T}=\mathfrak{T}^{\top}+\mathfrak{T}^{\perp}\right)$ : structure

$$
g^{\prime}:=g^{\top}+u^{2} g^{\perp}, \quad \mathfrak{T}^{\prime}:=u^{2} \mathfrak{T}^{\top}+\mathfrak{T}^{\perp},
$$

где $g^{\perp}(X, Y):=g\left(X^{\perp}, Y^{\perp}\right), \mathfrak{T}^{\top}(X, Y)=(\mathfrak{T}(X, Y))^{\top}$ и т. д.

Задача 1. Пусть $(M, g, \mathfrak{T})$ - расслоенное метрически-аффинное пространство. Найти $\mathcal{D}$-конформную структуру $\left(g^{\prime}, \mathfrak{T}^{\prime}\right)$, для которой $\overline{\mathrm{S}}_{\text {mix }}^{\prime}=n \Phi$ и функция $\Phi \in C^{\infty}(M)$ постоянна на листах.

Пусть $F$ - замкнутый лист слоения $\mathcal{F}$. Тогда оператор Шредингера

$$
\mathcal{H}=-\Delta^{\top}-\beta \text { id }
$$

имеет чисто точечный спектр $\lambda_{0} \leqslant \ldots \leqslant \lambda_{j} \rightarrow \infty$, причем наименьшее собственное значение $\lambda_{0} \in$ $[-\max \beta,-\min \beta]$ является простым; отвечающая ему собственная функция $e_{0}>0$ называется основным состоянием.

Пример 1. Метрически-аффинное дважды скрученное произведение $B \times{ }_{(v, u)} F$ метрическиаффинных многообразий $\left(B^{p}, g^{\top}, \mathfrak{T}^{\top}\right)$ и $\left(F^{n}, g^{\perp}, \mathfrak{T}^{\perp}\right)$ определяется как произведение $M=B \times F$, причем

$$
g=v^{2} g^{\top}+u^{2} g^{\perp}, \quad \mathfrak{T}=u^{2} \mathfrak{T}^{\top}+v^{2} \mathfrak{T}^{\perp},
$$

где $u, v>0$ - гладкие функции скручивания. Если перемножаемые многообразия являются пространствами Римана-Картана, то таковым является и $B \times_{(v, u)} F$. Листы пространства $B \times\{y\}$ и слои $\{x\} \times F$-вполне омбилические многообразия.

Пусть $\operatorname{Tr}\left(\mathfrak{T}^{\top}\right)=0$. Рассмотрим спектральную задачу $\mathcal{H}(u)=\left(\overline{\mathrm{S}}_{\mathrm{mix}} / n\right) u$, где

$$
\beta:=\frac{p}{n}\left[\frac{\Delta^{\perp} v}{v}-v\left\langle\operatorname{Tr}\left(\mathfrak{T}^{\perp}\right), \nabla v\right\rangle\right] .
$$

Пусть $B$ замкнуто и $v>0$ фиксировано. Как было указано выше, для $B \times_{\left(v, e_{0}\right)} F$ (т.е. $u=e_{0}$ ) функция $\overline{\mathrm{S}}_{\mathrm{mix}}=n \lambda_{0}$ постоянна на листах.

Свойство гармоничности $(\widetilde{H}=0)$ слоения $\mathcal{F}$ инвариантно относительно (4), поскольку

$$
\tilde{h}^{\prime}=u^{-2} \tilde{h}, \quad \widetilde{H}^{\prime}=u^{-2} \widetilde{H} .
$$

Предложение 2. При указанных выше условиях, $\widetilde{H}=0, \operatorname{Tr}^{\top} \mathfrak{T}=0=\operatorname{Tr}^{\perp} \mathfrak{T}$, имеет место следующее эллиптическое уравнение для и:

$$
\mathcal{H}(u)=\Psi_{1} u^{-1}-\Psi_{2} u^{-3}+\Psi_{3} u^{3},
$$

где

$$
n \Psi_{1}=\|\tilde{h}\|^{2}-b_{\mathfrak{T}}, \quad n \Psi_{2}=\|T\|^{2}, \quad n \Psi_{3}=a_{\mathfrak{T}}, \quad \beta=\Psi_{2}-\Psi_{1}-\Psi_{3}-\frac{\overline{\mathrm{S}}_{\mathrm{mix}}}{n}+\Phi, \quad \overline{\mathrm{S}}_{\text {mix }}^{\prime}=n \Phi
$$



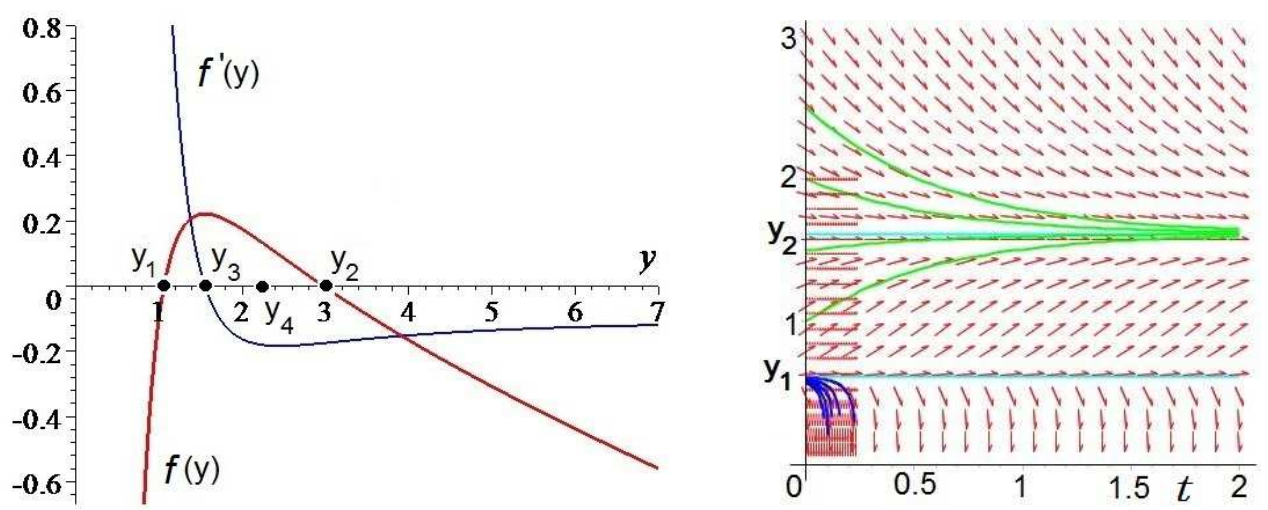

Рис. 1. Графики функций $f$ и $f^{\prime}$, случай $\Psi_{3}=0, \beta<0,4|\beta| \Psi_{2}<\Psi_{1}^{2}: y_{2}$ является устойчивым решением (аттрактором), а $y_{1}$ - неустойчивым.

(после $\mathcal{D}$-конформного преобразования) и

$$
\begin{gathered}
a_{\mathfrak{T}}=\sum_{i, a} \varepsilon_{i} \varepsilon_{a}\left(\left\langle\mathfrak{T}_{a a}^{\top}, \mathfrak{T}_{i i}^{\top}\right\rangle-\left\langle\mathfrak{T}_{i a}^{\top}, \mathfrak{T}_{a i}^{\top}\right\rangle\right), \\
b_{\mathfrak{T}}=-\sum_{i, a} \varepsilon_{i} \varepsilon_{a}\left\langle T\left(\mathfrak{T}_{i} E_{a}+\mathfrak{T}_{a} \mathcal{E}_{i}, \mathcal{E}_{i}\right), E_{a}\right\rangle .
\end{gathered}
$$

Свяжем с (5) следующую задачу Коши для функции $u(x, t)>0$ :

$$
\partial_{t} u+\mathcal{H}(u)=\Psi_{1} u^{-1}-\Psi_{2} u^{-3}+\Psi_{3} u^{3}, \quad u(\cdot, 0)=u_{0}>0 .
$$

Предположим, что решение $u^{*}$ существует и единственно; тогда оно является гладким на $M$, если предположить, что $\mathcal{F}$ определено ориентируемым расслоением $\pi: M \rightarrow B$.

Основная теорема (см. $[18,19])$. Пусть $H=0=\tilde{H}$. Тогда задача 1 имеет гладкое решение: функиия $\Phi$ постоянна на листах и $и>0$ на М. При выполнении некоторых условий для любой такой функции $\Phi$ решение и единственно в подходящей области.

Лемма 1. Пусть $\beta, \Psi_{1} u \Psi_{2}, \Psi_{3}$ - постоянные. Тогда $u=y(t)$, а задача (6) сводится к следующей задаче Коши для обыкновенного дифференииального уравнения:

$$
y^{\prime}=f(y):=\beta y+\Psi_{1} y^{-1}-\Psi_{2} y^{-3}+\Psi_{3} y^{3}, \quad y(0)=y_{0}>0 .
$$

Существуют три случая, в которых задача (7) имеет точечный аттрактор:

(i) $\Psi_{3}=0, \Psi_{1}>0, \Psi_{2} \geqslant 0, \lambda_{0}>0$ (например, $\bar{\nabla}=\nabla$; см. puс. 1 );

(ii) $\Psi_{3}<0, \Psi_{1}<0, \Psi_{2}>0, \lambda_{0}<0$;

(iii) $\Psi_{3}>0, \Psi_{1}>0, \Psi_{2}>0, \lambda_{0}>0$.

Поставляя $u=e_{0} w$ в (6), получаем задачу Коши

$$
\partial_{t} w=\Delta w+2\left\langle\nabla \log e_{0}, \nabla w\right\rangle+\tilde{f}, \quad w(\cdot, 0)=\frac{u_{0}}{e_{0}},
$$

где

$$
\tilde{f}=-\lambda_{0} w+\left(\Psi_{1} e_{0}^{-2}\right) w^{-1}-\left(\Psi_{2} e_{0}^{-4}\right) w^{-3}+\left(\Psi_{3} e_{0}^{2}\right) w^{3} .
$$

Из (8) получаем дифференциальные неравенства

$$
\phi_{-}(w) \leqslant \partial_{t} w-\Delta w-2\left\langle\nabla \log e_{0}, \nabla w\right\rangle \leqslant \phi_{+}(w),
$$

где функции $\phi_{-}$и $\phi_{+}$определяются для каждого случая отдельно. Будем искать устойчивые стационарные решения задачи (8), опираясь на приниип максимума для уравнения теплопрврдности с постоянными коэффициентами. Положим $\Psi_{3} \equiv 0$ (см. случай 1 ) и

$$
\Psi_{k}^{+}=\max _{F}\left(\left|\Psi_{k}\right| e_{0}^{-2 k}\right), \quad \Psi_{k}^{-}=\min _{F}\left(\left|\Psi_{k}\right| e_{0}^{-2 k}\right) .
$$


Тогда функции $\phi_{-}$и $\phi_{+}$в $(9)$ имеют вид $\phi_{ \pm}(y)=P_{\phi_{ \pm}}\left(y^{2}\right) / y^{3}$, где $P_{\phi_{ \pm}}(z)=-\lambda_{0} z^{2}+\Psi_{1}^{\mp} z-\Psi_{2}^{ \pm}$. Обозначим через $y_{i}^{ \pm}$вещественные нули функций $\phi_{ \pm}$и положим $\mathcal{U}_{1}=\left\{\tilde{u} \in C(F): \tilde{u} / e_{0}>y_{1}^{-}\right\}$.

Теорема 1. Пусть $\Psi_{3} \equiv 0, \Psi_{1}>0 u \Psi_{2}>0$. Если $0<\lambda_{0}<\left(\Psi_{1}^{-}\right)^{2} /\left(4 \Psi_{2}^{+}\right)$, то справедливъ неравенства $y_{1}^{+}<y_{1}^{-}<y_{3}^{-}<y_{2}^{-}<y_{2}^{+}$и эллиптическое уравнение

$$
\mathcal{H}(u)=\Psi_{1} u^{-1}-\Psi_{2} u^{-3}, \quad \mathcal{H}=-\Delta-\beta \mathrm{id},
$$

имеет в пространстве $\mathcal{U}_{1} \cap C^{\infty}(F)$ единственное решение $u_{*}$, удовлетворяющее условию $y_{1}^{-} \leqslant$ $u_{*} / e_{0} \leqslant y_{1}^{+}$. Кроме того, $u_{*}=\lim _{t \rightarrow \infty} u(\cdot, t)$ экспоненциально быстро относительно $C$-нормы, где и - решение параболической задачи

$$
\partial_{t} u+\mathcal{H}(u)=\Psi_{1} u^{-1}-\Psi_{2} u^{-3}, \quad u(\cdot, 0)=u_{0} \in \mathcal{U}_{1} .
$$

Остальные два случая (с $\left.\Psi_{3} \neq 0\right)$ анализируются аналогично (см. [19]).

4. Вариации функционалов на слоениях. Действие Эйнштейна-Гильберта определяется следующим образом:

$$
J:(g, \mathfrak{T}) \rightarrow \int_{M}\left\{\frac{1}{2 \mathfrak{a}}(\overline{\mathrm{S}}-2 \Lambda)+\mathcal{L}\right\} d \operatorname{vol}_{g} .
$$

Если $g$ изменяется, то уравнения Эйлера-Лагранжа приводят к уравнению Эйнштейна

тензор энергии-импульса имеет вид

$$
\overline{\operatorname{Ric}}-\frac{1}{2} \overline{\mathrm{S}} \cdot g+\Lambda g=\mathfrak{a} \Theta,
$$

$$
\Theta_{\mu \nu}=-2 \frac{\partial \mathcal{L}}{\partial g^{\mu \nu}}+g_{\mu \nu} \mathcal{L}
$$

где $\Lambda$ - космологическая постоянная, $\mathcal{L}$-лагранжиан, описывающий материю, $\mathfrak{a}$ - постоянная взаимодействия. Если $\mathfrak{T}$ изменяется, то уравнения Эйлера-Лагранжа приводят к соотношению между кручением и спином материальных полей (теория относительности ЭйнштейнаКартана; см., например, [1]):

$$
\mathcal{S}_{\cdot j k}^{i}+\delta_{j}^{i} \mathcal{S}_{\cdot k a}^{a}+\delta_{k}^{i} \mathcal{S}_{\cdot j a}^{a}=\mathfrak{a} s_{\cdot j k}^{i} .
$$

Смешанное действие Эйнштейна-Гилъберта (см. [3]) - это аналог действия ЭйнштейнаГильберта, в котором скалярная кривизна заменена величиной $\mathrm{S}_{\mathrm{mix}}$ :

$$
J_{\mathcal{D}, \Omega}:(g, \mathfrak{T}) \mapsto \int_{\Omega}\left\{\frac{1}{2 \mathfrak{a}}\left(\overline{\mathrm{S}}_{\text {mix }}-2 \Lambda\right)+\mathcal{L}\right\} d \operatorname{vol}_{g} .
$$

Физический смысл (11) обсуждается в [3].

Будем использовать финитные (т.е. имеющие компактный носитель) вариации $\left\{g_{t} \in \operatorname{Riem}(M)\right.$ : $|t|<\varepsilon\}$ метрики $g_{0}=g$. Рассмотрим ортогональное разложение тензора $B=\partial_{t} g_{t} \in \operatorname{Sym}^{2}(M)$ вида $B=B^{\perp}+B^{\vee}+B^{\top}$, где компонентами являются симметричные тензоры

$$
\begin{gathered}
B^{\top}(X, Y):=B\left(X^{\top}, Y^{\top}\right), \quad B^{\perp}(X, Y):=B\left(X^{\perp}, Y^{\perp}\right), \\
B^{\vee}(X, Y):=\frac{1}{2}\left(B\left(X^{\top}, Y^{\perp}\right)+B\left(X^{\perp}, Y^{\top}\right)\right) .
\end{gathered}
$$

Определение 1. Семейство $g_{t},|t|<\varepsilon$, называется $g^{\pitchfork}$-вариацией для $(\widetilde{\mathcal{D}}, \mathcal{D})$, если метрика на $\widetilde{\mathcal{D}}$ сохраняется:

$$
g_{t}(X, Y)=g(X, Y), \quad X, Y \in \mathfrak{X}_{\widetilde{\mathcal{D}}}, \quad|t|<\varepsilon .
$$

Если распределения $\widetilde{\mathcal{D}}$ и $\mathcal{D}$ остаются ортогональными, то $g_{t}$ называется адаптированной вариаиией, и мы имеем $B^{\vee}=0$; кроме того, если изменяется только метрика на $\widetilde{\mathcal{D}}$, т.е.

то вариация называется $g^{\top}$-вариацией.

$$
\left.g_{t}(X, Y)=g(X, Y), \quad X, Y \in \mathfrak{X}_{\mathcal{D}}\right),
$$

Вместо вычислений в координатах будем использовать локальные ортонормированные реперы. 
Лемма 2. Предположим, что локалъный $(\widetilde{\mathcal{D}}, \mathcal{D})$-адаптированный и ортонормированный при $t=0$ репер $\left\{E_{a}(t), \mathcal{E}_{i}(t)\right\}$ изменяется под действием $g_{t} \in \operatorname{Riem}(M),|t|<\varepsilon$, согласно уравнениям

$$
\partial_{t} E_{a}=-\frac{1}{2} B_{t}^{\sharp}\left(E_{a}\right)^{\top}, \quad \partial_{t} \mathcal{E}_{i}=-\frac{1}{2} B_{t}^{\sharp}\left(\mathcal{E}_{i}\right)^{\perp}-B_{t}^{\sharp}\left(\mathcal{E}_{i}\right)^{\top} .
$$

Тогда при всех $t$ репер $\left\{E_{a}(t), \mathcal{E}_{i}(t)\right\}$ является $g_{t}$-ортонормированным репером, адаптированным $\kappa \widetilde{\mathcal{D}}$, m.е. $\left\{E_{a}(t)\right\}$ принадлежит $\widetilde{\mathcal{D}}, a\left\{\mathcal{E}_{i}(t)\right\}$ принадлежит $\mathcal{D}_{t}$.

Для любых $(0,2)$-тензоров $P, Q$ и $S$ определим тензор $\Upsilon_{P, Q}$ следующим образом:

$$
\left\langle\Upsilon_{P, Q}, S\right\rangle=\sum_{\lambda, \nu} \varepsilon_{\lambda} \varepsilon_{\nu}\left[S\left(P\left(e_{\lambda}, e_{\nu}\right), Q\left(e_{\lambda}, e_{\nu}\right)\right)+S\left(Q\left(e_{\lambda}, e_{\nu}\right), P\left(e_{\lambda}, e_{\nu}\right)\right)\right] .
$$

Введем также тензоры

$$
\mathcal{K}=\sum_{i} \varepsilon_{i}\left[T_{i}^{\sharp}, A_{i}\right], \quad \operatorname{div} h=\sum_{\nu} \varepsilon_{\nu} g\left(\nabla_{\nu} h, e_{\nu}\right) .
$$

и симметричные $(0,2)$-тензоры $\alpha, \theta, \delta_{Z}$

$$
\begin{gathered}
2 \alpha(X, Y)=A_{X^{\perp}}\left(Y^{\top}\right)+A_{Y^{\perp}}\left(X^{\top}\right), \quad 2 \theta(X, Y)=T_{X^{\perp}}^{\sharp}\left(Y^{\top}\right)+T_{Y^{\perp}}^{\sharp}\left(X^{\top}\right), \\
2 \delta_{Z}(X, Y)=\left\langle\nabla_{X^{\top}} Z, Y^{\perp}\right\rangle+\left\langle\nabla_{Y^{\top}} Z, X^{\perp}\right\rangle .
\end{gathered}
$$

Предложение 3. Для любой вариации $g_{t}$ метрики, удовлетворяющей условиям $\dot{g}=B u$ $\mathfrak{T}=0$ имеем

$$
\begin{aligned}
& \partial_{t}\|\tilde{h}\|^{2}=\left\langle\operatorname{div} \tilde{h}-4 \Upsilon_{\tilde{\alpha}, \theta}+\widetilde{\mathcal{K}}^{b}-\frac{1}{2} \Upsilon_{\tilde{h}, \tilde{h}}, B\right\rangle-\operatorname{div}\langle\tilde{h}, B\rangle, \\
& \partial_{t}\|\widetilde{H}\|^{2}=\left\langle(\operatorname{div} \widetilde{H}) g^{\perp}+4\langle\theta, \widetilde{H}\rangle-\widetilde{H}^{b} \otimes \widetilde{H}^{b}, B\right\rangle-\operatorname{div}\left(\left(\operatorname{Tr}_{\mathcal{D}} B^{\sharp}\right) \widetilde{H}\right), \\
& \partial_{t}\|h\|^{2}=\left\langle\operatorname{div} h+\mathcal{K}^{b}-2(\operatorname{div} \alpha)_{\mid \vee}-2 \Upsilon_{\alpha, \tilde{\alpha}+\tilde{\theta}}-\frac{1}{2} \Upsilon_{h, h}, B\right\rangle+\operatorname{div}\langle 2 \alpha-h, B\rangle, \\
& \partial_{t}\|H\|^{2}=\left\langle 2\langle\tilde{\theta}-\tilde{\alpha}, H\rangle+2 \operatorname{Sym}\left(H^{b} \otimes \widetilde{H}^{b}\right)-2 \delta_{H}+(\operatorname{div} H) g^{\top}-H^{b} \otimes H^{b}, B\right\rangle+ \\
& \quad+2 \operatorname{div}\left(\left(B^{\sharp} H\right)^{\top}-\left(\operatorname{Tr}_{\widetilde{\mathcal{D}}} B^{\sharp}\right) H\right), \\
& \partial_{t}\|\tilde{T}\|^{2}=\left\langle 2 \widetilde{\mathcal{T}}^{b}+2 \Upsilon_{\tilde{\theta}, \theta-\alpha}-2(\operatorname{div} \tilde{\theta})_{\mid \vee}+\frac{1}{2} \Upsilon_{\tilde{T}, \tilde{T}}, B\right\rangle+2 \operatorname{div}\langle\tilde{\theta}, B\rangle, \\
& \partial_{t}\|T\|^{2}=\left\langle\frac{1}{2} \Upsilon_{T, T}+2 \mathcal{T}^{\downarrow}, B\right\rangle .
\end{aligned}
$$

Эти формулы применимы к различным функционалам на псевдоримановом многообразии с распределением.

Определение 2. Смешанный тензор Риччи $\operatorname{Ric}_{\mathcal{D}}$ метрики $g \in \operatorname{Riem}(M, \widetilde{\mathcal{D}}, \mathcal{D})$ - это симметричный $(0,2)$-тензор

$$
\operatorname{Ric}_{\mathcal{D}}=\left(\operatorname{Ric}_{\mathcal{D}}\right)^{\perp}+\left(\operatorname{Ric}_{\mathcal{D}}\right)^{\vee}+\left(\operatorname{Ric}_{\mathcal{D}}\right)^{\top}
$$

где

$$
\begin{gathered}
\left(\operatorname{Ric}_{\mathcal{D}}\right)^{\perp}=\operatorname{div} \tilde{h}+\widetilde{\mathcal{K}}^{b}-2 \widetilde{\mathcal{T}}^{b}+H^{b} \otimes H^{b}-\frac{1}{2} \Upsilon_{h, h}-\frac{1}{2} \Upsilon_{T, T}-\frac{n-1}{p+n-2} \operatorname{div}(\widetilde{H}-H) g^{\perp}, \\
\frac{1}{2}\left(\operatorname{Ric}_{\mathcal{D}}\right)^{\vee}=\delta_{H}+(\operatorname{div}(\tilde{\theta}-\alpha))_{\mid \vee}-2\langle\theta, \widetilde{H}\rangle-\operatorname{Sym}\left(H^{b} \otimes \widetilde{H}^{b}\right)-\langle\tilde{\theta}-\tilde{\alpha}, H\rangle-2 \Upsilon_{\tilde{\alpha}, \theta}-\Upsilon_{\alpha, \tilde{\alpha}}-\Upsilon_{\tilde{\theta}, \theta}, \\
\left(\operatorname{Ric}_{\mathcal{D}}\right)^{\top}=\text { двойственный к }\left(\operatorname{Ric}_{\mathcal{D}}\right)^{\perp} .
\end{gathered}
$$

Теорема 2. Уравнение Эйлера-Лагранжа для действия (11) имеет вид

$$
\operatorname{Ric}_{\mathcal{D}}-(1 / 2) \mathrm{S}_{\mathcal{D}} \cdot g+\Lambda g=\mathfrak{a} \Theta
$$


(ср. с уравнением Эйнштейна), а танзор Риччи и скаларная кривизна заменяются на кривизну muпа Риччи $\operatorname{Ric}_{\mathcal{D}}$ и ее след

$$
\mathrm{S}_{\mathcal{D}}=\mathrm{S}_{\mathrm{mix}}+\frac{p-n}{n+p-2} \operatorname{div}(H-\tilde{H}) .
$$

Пример 2. Пространство-время $\left(M^{4}, g\right)$ с времениподобным единичным векторным полем $N$. Для распределения $\widetilde{\mathcal{D}}$, натянутого на $N$, положим $\tau_{i}=\operatorname{Tr}\left(\left(A_{N}\right)^{i}\right)$. Тогда (см. [3])

$$
\begin{aligned}
& \operatorname{Ric}_{\mathcal{D} \mid \mathcal{D} \times \mathcal{D}}=\nabla_{N} h_{s c}-\tau_{1} h_{s c}-\varepsilon_{N}\left(2\left(T_{N}^{\sharp}\right)^{2}+\left[T_{N}^{\sharp}, A_{N}\right]\right)^{b}, \\
& \operatorname{Ric}_{\mathcal{D}}(\cdot, N)_{\mid \mathcal{D}}=\operatorname{div}^{\perp}\left(T_{N}^{\sharp}\right)+2\left(T_{N}^{\sharp} H\right)^{b}, \\
& \operatorname{Ric}_{\mathcal{D}}(N, N)=\varepsilon_{N}\left(N\left(\tau_{1}\right)-\tau_{2}\right)-\|T\|^{2}, \\
& \mathrm{~S}_{\mathcal{D}}=\varepsilon_{N} \operatorname{Ric}_{N, N}+\operatorname{div}\left(\varepsilon_{N} \tau_{1} N-H\right) .
\end{aligned}
$$

Замечание 1. Можно рассмотреть возмущенное действие Эйнштейна-Гильберта

$$
J_{\varepsilon}:(g, \mathfrak{T}) \mapsto \int_{\Omega}\left\{\frac{1}{2 \mathfrak{a}}\left(S+\varepsilon S_{\mathrm{mix}}-2 \Lambda\right)+\mathcal{L}\right\} d \operatorname{vol}_{g}
$$

где $\varepsilon \in \mathbb{R}$; ему соответствует возмущенное уравнение Эйнштейна

$$
\operatorname{Ric}(\varepsilon)+(1 / 2) S(\varepsilon)+\Lambda g=\mathfrak{a} \Theta,
$$

где

$$
\operatorname{Ric}(\varepsilon):=\operatorname{Ric}+\varepsilon \operatorname{Ric}_{\mathcal{D}}, \quad S(\varepsilon)=\operatorname{Tr}_{g} \operatorname{Ric}(\varepsilon) .
$$

Возмущенное уравнение можно использовать для моделирования в общей теории относительности. В последующих статьях автор планирует рассмотреть теорию Эйнштейна-Картана, в основе которой лежит уравнение Эйлера-Лагранжа для действия (11) с переменной $\mathfrak{T}$, по аналогии c (10).

Далее рассмотрим приложения к контактным структурам и 3-структурам Сасаки. Происхождение контактных многообразий связано с естественными слоениями, порожденными полем Риба. Они допускают также ассоциированные метрики (вообще говоря, неединственные) с хорошо изученными свойствами (см. [5]). Покажем, что такие метрики и сасакиевы 3-структуры являются естественным источником метрик, критических для действия

$$
J_{\text {mix }, \widetilde{\mathcal{D}}}: g \rightarrow \int_{M} \mathrm{~S}_{\text {mix }}(g) d \operatorname{vol}_{g} .
$$

Предложение 4. Любая $K$-контактная метрика g, т.е. метрика, для которой $N$ - поле Киллинга, является критической для действия (12) по отношению $к$ сохраняюшим обгем $g^{\pitchfork}$ и $g^{\top}$-вариачиям.

Напомним (см. [5, с. 24]), что контактная структура называется регулярной, если $\xi$ регулярно как векторное поле, т.е. каждая точка многообразия обладает окрестностью, через которую каждая интегральная кривая поля $\xi$ проходит единожды. Следующая теорема является обобщением [5, Theorem 10.12].

Теорема 3. Ассочиированнал (с данным $\eta$ ) метрика g на компактном регулярном контактном многообразии $(M, \eta)$ является критической для действия (12) среди метрик, ассочиированных с $\eta$, тогда и толъко тогда, когда она К-контактна.

Предложение 5. Метрика 3-структуры Сасаки на многообразии $M$ является критической для действия (12) (әде распределение $\widetilde{\mathcal{D}}$ натлнуто на характеристические векторные поля) по отношению к сохраняющим обгем $g^{\dagger}$-вариачий $и g^{\top}$-вариаций. 


\section{СПИСОК ЛИТЕРАТУРЫ}

1. Aldrovandi R., Pereira J. G. Teleparallel Gravity. An Iintroduction. - Dordrecht: Springer, 2013.

2. Aubin T. Some Nonlinear Problems in Riemannian Geometry. - Springer, 1998.

3. Barletta E., Dragomir S., Rovenski V., Soret M. Mixed gravitational field equations on globally hyperbolic spacetimes// Class. Quant. Gravity. - 2013. - 30, № 8. - 085015.

4. Bernal A. N., Sánchez M. Smoothness of time functions and the metric splitting of globally hyperbolic spacetimes// Commun. Math. Phys. - 2005. - 257. - P. 43-50.

5. Blair D. Riemannian Geometry of Contact and Symplectic Manifolds. - Springer, 2010.

6. Candel A., Conlon L. Foliations. - Providence, Rhode Island: Am. Math. Soc., 2003.

7. Kazdan J. L., Warner F. W. Curvature functions for compact 2-manifolds// Ann. Math. - 1974. - 99. P. $14-47$.

8. Lużyńczyk M., Walczak P. New integral formulae for two complementary orthogonal distributions on Riemannian manifolds// Ann. Glob. Anal. Geom. - 2015. - 48. - P. 195-209.

9. Rovenski V. Foliations on Riemannian Manifolds and Submanifolds. - Basel: Birkhäuser, 1998.

10. Rovenski $V$. Integral formulae for a Riemannian manifold with two orthogonal distributions// Centr. Eur. J. Math. - 2011. - 9, № 3. - P. 558-577.

11. Rovenski V. On solutions to equations with partial Ricci curvature// J. Geom. Phys. - 2014. - 86. P. $370-382$.

12. Rovenski V. Einstein-Hilbert-type action on spacetimes// Publ. Inst. Math. - 2018. - 103 (117). - P. 199 210.

13. Rovenski $V$. Integral formulas for a metric-affine manifold with two complementary orthogonal distributions// Global J. Adv. Res. Class. Modern Geom. - 2017. - 6, № 1. - P. 7-19.

14. Rovenski V., Walczak P. Integral formulae on foliated symmetric spaces// Math. Ann. - 2012. - 352, № 1. - P. 223-237.

15. Rovenski V., Walczak P. Topics in Extrinsic Geometry of Codimension-One Foliations. - Springer, 2011.

16. Rovenski $V$., Zawadzki T. The Einstein-Hilbert type action on pseudo-Riemannian almost product manifolds// J. Math. Phys. Anal. Geom. - 2019. - 15, № 1. - P. 86-121.

17. Rovenski V., Zawadzki T. Variations of the total mixed scalar curvature of a distribution// Ann. Glob. Anal. Geom. - 2018. - 54. - P. 87-122.

18. Rovenski V., Zelenko L. The mixed Yamabe problem for harmonic foliations// Eur. J. Math. - 2015. - 1 . - P. 503-533.

19. Rovenski V., Zelenko L. Prescribing mixed scalar curvature of foliated Riemann-Cartan spaces// J. Geom. Phys. - 2018. - 126. - P. 42-67.

20. Stepanov S., Mikeš J. Liouvile-type theorems for some classes of Riemannian almost product manifolds and for special mappings of Riemannian manifolds// Differ. Geom. Appl. - 2017. - 54. - P. 111-121.

21. Walczak P. An integral formula for a Riemannian manifold with two orthogonal complementary distributions// Colloq. Math. - 1990. - 58. - P. 243-252.

Ровенский Владимир Юзефович

University of Haifa, Israel

E-mail: vrovenski@univ.haifa.ac.il 